\title{
Registrar para avanzar
}

\author{
Laia Matarranz Torres \\ Vocal de la Junta Directiva de SEFAC y coordinadora de la comisión de Nuevas Tecnologías. Coordinadora del proyecto SEFAC e_XPERT.
}

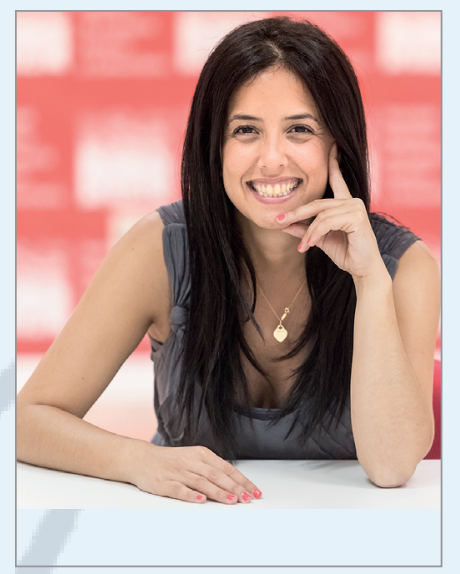

Laia Matarranz Torres
El farmacéutico comunitario es un profesional sanitario con un enorme potencial para mejorar no solo el uso de los medicamentos (detectando, por ejemplo, resultados negativos de la medicación), sino también la prevención de enfermedades y la promoción de la salud pública. Sin duda, el desarrollo y el impulso que están teniendo los servicios profesionales farmacéuticos (SPF) desde hace años pueden contribuir decisivamente a que este potencial se consolide de una forma definitiva, obteniendo el reconocimiento del conjunto del sistema sanitario, incluyendo tanto a otros profesionales de la salud (médicos, enfermería, medicina especializada ) como a la Administración y, por supuesto, a los propios pacientes beneficiarios de estos servicios. Es cierto que la figura del farmacéutico y su ejercicio sanitario han estado tradicionalmente bien considerados por la población, pero no es menos verdad que la integración de la actividad asistencial de la farmacia comunitaria en el Sistema Nacional de Salud (SNS) es, cuanto menos mejorable, y que, incluso, algunas manifestaciones ponen en entredicho de vez en cuando la labor científico-asistencial de las farmacias o generan dudas sobre su capacidad para formar parte de pleno derecho de los equipos de salud que deben trabajar de forma coordinado en la mejora de la atención sanitaria de los pacientes. Es el caso de la salida del canal de la farmacia comunitaria de medicamentos innovadores o la imposibilidad de que las farmacias puedan intervenir en algunas comunidades autónomas en políticas de prevención como los cribados. Además, algunos trabajos publicados, como por ejemplo el estudio Refcom. La realidad de la farmacia comunitaria en España, promovido en su día por SEFAC, reconocía la falta de integración de la farmacia comunitaria en las estrategias de salud del sistema sanitario, ya que el 49\% de la población participante en este estudio consideraba que los conocimientos de los farmacéuticos no se aprovechan lo suficiente y solo el 51\% de los encuestados creía que el farmacéutico comunitario estaba bien integrado en el sistema sanitario.

Estos datos revelan que hay que seguir trabajando con intensidad para que la formación y la proximidad que los farmacéuticos poseemos con la población en general y los pacientes en particular puedan dar unos mejores réditos al SNS. La provisión de servicios profesionales farmacéuticos asistenciales, protocolizados, basados en el conocimiento, consensuados y/o coordinados en el marco de un trabajo interdisciplinar debe ser el camino para conseguir el objetivo de que el farmacéutico comunitario sea incorporado en plenitud a todas las estrategias sanitarias y sociosanitarias en las que se quiera mejorar la prevención de la enfermedad y el uso adecuado de los medicamentos.

Pero, además, hace falta completar ese camino con la capacidad para generar evidencia científica sobre el impacto en la salud de los SPF y su eficiencia para el sistema. Esto pasa por el ineludible registro de los datos que se generan en la farmacia con su actividad asistencial. De poco vale glosar el potencial sanitario de la farmacia, su proximidad, su cercanía o la calidad de su formación si no se respalda con datos que avalen ante el resto de agentes el valor imprescindible de la farmacia comunitaria. ¿Significa esto que no hay datos que apoyen la calidad de las intervenciones farmacéuticas en salud? En absoluto, muchos de los artículos publicados en FARMACÉUTICOS COMUNITARIOS y otras revista así lo demuestran $\mathrm{y}$, de hecho, hay distintos estudios como, por ejemplo, $D$-VALOR (dispensación), I-VALOR (indicación), Consigue (seguimiento farmacoterapéutico) o Revisa (revisión del uso de los medicamentos) que han aportado ya evidencias del valor que pueden tener los SPF, por no hablar de otras investigaciones en el plano internacional. Sin embargo, la cultura del registro de datos en el día a día de las farmacias, por distintos factores (entre ellos la existencia de una gran carga burocrática), es evidente y hay que tratar de revertir de forma colectiva 
esta situación para que se incorpore a la práctica diaria de los farmacéuticos que desarrollan servicios profesionales y la recopilación de datos sea mucho más numerosa de lo que es en la actualidad. Si tenemos en cuenta que más de dos millones de personas pasan a diario por las farmacias en España, la capacidad para recabar datos de forma asequible es muy grande y puede dar mucho más de sí.

En SEFAC, como sociedad científica, creemos firmemente en la trascendencia de generar esta evidencia mediante la cultura del registro como vehículo para seguir avanzando y conseguir revalorizar la labor asistencial de los farmacéuticos comunitarios como profesionales de la salud de primer orden. Así se ha venido poniendo de manifiesto en los últimos años y, más concretamente, en la serie de jornadas que SEFAC ha venido celebrando desde principios de año en sus distintas delegaciones con el inequívoco lema de Viviendo la farmacia de servicios profesionales. De ahí que los distintos programas de capacitación en servicios profesionales siempre incluyan una fase final de registro de casos clínicos con el objetivo de generar esa evidencia científica necesaria. La prueba más palpable de esta filosofía es el actual estudio Impacto del servicio farmacéutico de cesación tabáquica en la farmacia comunitaria, que en estos momentos está en marcha y que incluirá a mil pacientes, lo que le convertirá en el mayor estudio realizado en farmacia comunitaria sobre cesación tabáquica. Con los resultados no solo se persigue demostrar la eficacia de la intervención farmacéutica para ayudar a dejar de fumar; también se busca la eficiencia y demostrar ante la Administración que los servicios profesionales farmacéuticos pueden ser de gran ayuda en la sostenibilidad del SNS. En definitiva, el objetivo es dejar constancia de que un buen catálogo de SPF es eficaz, eficiente y rentable, lo que debería facilitar la creación de una cartera retribui- da de servicios profesionales farmacéuticos asistenciales por parte del SNS.

Pero SEFAC también es consciente de que implantar esta filosofía de registro continuo requiere herramientas facilitadoras. Por este motivo se ha creado SEFAC e_XPERT, una plataforma online de gestión de servicios profesionales farmacéuticos (www.sefacexpert.org), que también se ha venido presentando en las jornadas SEFAC de este año. El objetivo de esta plataforma no es otro que facilitar la ejecución de los SPF, desde el registro de datos de salud, seguimiento y evolución del paciente hasta la generación de informes de derivación al médico, pasando por la facturación del servicio. En SEFAC e_XPERT se incluyen en la actualidad módulos para el desarrollo de los servicios de medida y seguimiento de la presión arterial, cálculo del riesgo vascular y cesación tabáquica. Próximamente está previsto que se sumen otros como el de revisión del uso de los medicamentos, consejo nutricional, diabetes y Enfermedad Pulmonar Obstructiva Crónica (EPOC). También incluye una funcionalidad diseñada especialmente para su uso en el mostrador y destinada a registrar de forma rápida la entrada de datos personales y datos clave de salud de un paciente. Esto facilita tener una visión global del estado inicial de salud de una persona y poder derivar a un servicio profesional farmacéutico en caso de que fuera necesario.

Hay, por tanto, motivos más que suficientes y herramientas para que los farmacéuticos comunitarios asumamos la necesidad de que si queremos reivindicar con más fuerza nuestra labor sanitaria urge plasmarla en datos. Esto debe servirnos también para potenciar la investigación y la publicación de artículos científicos en atención farmacéutica y, más concretamente, en servicios profesionales farmacéuticos. Registrar para avanzar, esa es la clave. 